\title{
Lentivirus-mediated silencing of the PTC1 and PTC2 genes promotes recovery from spinal cord injury by activating the Hedgehog signaling pathway in a rat model
}

\author{
Ya-Dong Zhang ${ }^{1}$, Zhong-Sheng Zhu' ${ }^{1}$, Dong Zhang ${ }^{1}$, Zhen Zhang ${ }^{2}$, Bin $\mathrm{Ma}^{2}$, Shi-Chang Zhao and Feng Xue ${ }^{1}$
}

This study aimed to investigate the effect of Patched-1 (PTC1) and PTC2 silencing in a rat model, on Hedgehog (Hh) pathwaymediated recovery from spinal cord injury (SCl). An analytical emphasis on the relationship between the sonic hedgehog (Shh) pathway and nerve regeneration was explored. A total of 126 rats were divided into normal, sham, SCl, negative control (NC), PTC1-RNAi, PTC2-RNAi and PTC1/PTC2-RNAi groups. The Basso, Beattie and Bresnahan (BBB) scale was employed to assess hind limb motor function. Quantitative real-time polymerase chain reaction and western blotting were performed to examine the mRNA and protein levels of PTC1, PTC2, Shh, glioma-associated oncogene homolog 1 (Gli-1), Smo and Nestin. Tissue morphology was analyzed using immunohistochemistry, and immunofluorescent staining was conducted to detect neurofilament protein 200 (NF-200) and glial fibrillary acidic protein (GFAP). The PTC1/PTC2-RNAi group displayed higher BBB scores than the SCl and NC groups. Shh, Gli-1, Smo and Nestin expression levels were elevated in the PTC1/PTC2-RNAi group. PTC1 and PTC2 mRNA and protein expression was lower in the PTC1/PTC2-RNAi group than in the normal, sham and SCI groups. Among the seven groups, the PTC1/PTC2-RNAi group had the largest positive area of NF-200 staining, whereas the SCI group exhibited a larger GFAP-positive area than both the normal and the sham groups. The Shh pathway may provide new insights into therapeutic indications and regenerative recovery tools for the treatment of $\mathrm{SCl}$. Activation of the $\mathrm{Hh}$ signaling pathway by silencing PTC1 and PTC2 may reduce inflammation and may ultimately promote SCI recovery.

Experimental \& Molecular Medicine (2017) 49, e412; doi:10.1038/emm.2017.220; published online 15 December 2017

\section{INTRODUCTION}

Spinal cord injury (SCI) is a devastating medical condition characterized by motor, cardiac, bowel, sensory and bladder dysfunction. ${ }^{1}$ SCI is understood to progress in two stages: first through the mechanical disruption of various nervous tissues, followed by a serious perturbation of the local blood supply manifested by the secretion of pro-inflammatory mediators and neurotoxins from both resident and invading cells. ${ }^{2}$ Worldwide, more than 30000 new SCI cases occur each year, exhibiting relatively high morbidity, which requires long-term physical and medical care for SCI patients. ${ }^{3}$ A range of approaches have been adopted for treating SCI, and unfortunately, all have achieved only relatively limited success. ${ }^{4}$ Using various degenerative mechanisms, SCI not only gives rise to acute and progressive secondary disruption of local and distant nervous tissues, but it also causes a series of endogenous regenerative and neuro-protective reactions. ${ }^{5} \mathrm{~A}$ comprehensive investigation of such mechanisms may facilitate current and future SCI treatment approaches. It has been suggested that the Sonic hedgehog (Shh) signaling pathway may play a vital role in nerve regeneration after injury. Previous research has provided evidence alluding to the notion that nerve function is improved by Shh treatment in diabetic models of neuropathy. ${ }^{6,7}$ Thus, it has been hypothesized that the Shh signaling pathway may contribute to nerve regeneration after SCI and may promote the recovery of SCI patients.

The hedgehog $(\mathrm{Hh})$ signaling pathway is crucial for the patterning and growth of various tissues during embryonic

\footnotetext{
${ }^{1}$ Department of Orthopaedics, Shanghai Fengxian Central Hospital, South Campus of the Sixth People's Hospital Affiliated to Shanghai Jiao Tong University, Shanghai, People's Republic of China; '2Department of Spine Surgery, Shanghai East Hospital, School of Medicine, Tongji University, Shanghai, People's Republic of China and ${ }^{3}$ Department of Orthopedics, the Affiliated Sixth People's Hospital of Shanghai Jiao Tong University, Shanghai, People's Republic of China

Correspondence: Dr Y-D Zhang or Dr F Xue, Department of Orthopaedics, Shanghai Fengxian Central Hospital, Branch of the Sixth People's Hospital Affiliated to Shanghai Jiao Tong University, No. 6600 Nanfeng Road, Shanghai 201499, People's Republic of China.
}

E-mail: yadd_zz@163.com or fengff_xx@163.com

Received 12 February 2017; revised 2 June 2017; accepted 25 June 2017 
development. ${ }^{8}$ Both Desert (Dhh) and Indian hedgehog (Ihh) belong to the Hh family and are well-described ligands in this signaling pathway. ${ }^{9}$ Shh, a key protein involved in craniofacial morphogenesis, promotes cell proliferation during embryogenesis in a tissue-specific manner by regulating epithelialmesenchymal interactions. ${ }^{10}$ The Shh signaling pathway initiates the destruction of a large cytoplasmic protein complex and promotes the release of oncogene homolog (Gli) transcription factors. The Shh-Gli signaling pathway plays diverse roles in mammalian development, including the regulation of cell fate determination, cell proliferation, cell differentiation, and cell survival and patterning of the central nervous system. ${ }^{11}$ The Hh proteins secreted in the Hh signaling pathway interact with the membrane-bound receptors Patched-1 (PTC1) and Patched-2 (PTC2), relieving the patched-mediated repression of the signal transducer smoothened (Smo) and activating glioma-associated transcription factors (Gli-1, Gli-2 and Gli-3) and promoting the transcription of downstream genes such as Ptc1, Ptc2 and Gli1. ${ }^{12}$ Considering that PTC1 negatively regulates Hh signaling during embryonic development, ${ }^{13}$ the present study aimed to investigate whether PTC1 and PTC2 silencing could promote SCI recovery via the Hh signaling pathway.

\section{MATERIALS AND METHODS}

\section{Ethics statement}

The experiment was conducted with approval from the Ethics Committee of Shanghai Fengxian Central Hospital, Branch of the Sixth People's Hospital Affiliated to Shanghai Jiao Tong University. All components of the study were completed in strict accordance with all relevant regulations.

\section{Experimental animals}

A total of 126 specific-pathogen-free (SPF) male Wistar rats were selected for the purposes of the study (weight: 200-220 g; age: 7 weeks old; license number: SCXK Sichuan Province 2016; Beijing Huafukang Biotechnology, Beijing, China). The chosen rats were fed according to the standard procedures.

\section{Establishment of the SCI rat model}

The abdominal cavity of the rats was injected with $10 \%$ chloral hydrate anesthetic. Following identification of the spinal cord region (T7-T9 segment), a median incision (3-4 cm) was created, followed by an incision layer-by-layer incisions through the skin, subcutaneous tissues and fascia to separate the lateral muscular tissues and to expose the neural plates. A micro-rongeur (Friedman-Person, $14 \mathrm{~cm}-$ diameter, Fine Science Tools, Hunan Yuanxiang Bio-tech, Changsha, Hunan, China) was used to fracture the spinous process and neural plate to fully expose the spinal cord. After cord exposure, SCI rat models were established using a modified Allen's method. A stainless steel bar (diameter: $2.5 \mathrm{~mm}$; weight: $5.0 \mathrm{~g}$ ) was vertically released along a graduated tube from a height of $5 \mathrm{~cm}$ and bumped with a plastic bar (diameter: $3 \mathrm{~mm}$ ) at the bottom to damage the rat spinal cords, which were immediately weighed and sutured using 0 stitches. Successful establishment of the rat model was based on whether the spinal cord exhibited hemorrhage and edema, the lower limbs of the rats fluttered contractively, their tails swayed spastically and finally flaccid paralysis was manifested. ${ }^{14}$ After successful establishment of the SCI rat models, hemostasis by compression was performed using a gelatin sponge until no obvious bleeding was observed at the operative site. When the spinal cord was clearly exposed, the rats were fixed on an intravenous stand.

\section{Lentivirus-mediated RNAi targeting of PTC1 and PTC2}

Short-hairpin RNAs (shRNAs) targeting PTC1 and PTC2 were designed based on the PTC1 (no. AB000848.1) and PTC2 (no. AB000846.1) sequences from GenBank. Two siRNAs, siRNA-1PTC1 (positive-sense strand: $5^{\prime}$-acagttcgaccctttggaa- $3^{\prime}$; negative-sense strand: $5^{\prime}$-ttccaaagggtcgaactgt-3') and siRNA-2-PTC1 (positive-sense strand: $\quad 5^{\prime}$-gaatcccacagatcccgaa- $3^{\prime}$; negative-sense strand: $5^{\prime}$-ttcgggatctgtgggattc- $3^{\prime}$ ) were designed to target sequences starting at PTC1 nucleotides 121 and 181, respectively. Similarly, two siRNAs, siRNA-1-PTC2 (positive-sense strand: $5^{\prime}$-cctaaatccaatgcagctg-3'; negative-sense strand: $5^{\prime}$-cagctgcattggatttagg- $3^{\prime}$ ) and siRNA-2-PTC2 (positive-sense strand: $5^{\prime}$-aagagacgggttcccaaca- $3^{\prime}$; negative-sense strand: $5^{\prime}$-tgttgggaacccgtctctt- $3^{\prime}$ ) were designed to target sequences starting at PTC2 nucleotides 61 and 161 , respectively. The DNA oligonucleotides were synthesized using Sangon Biotech (Shanghai, China). The positive and negative single-stranded DNAs were annealed and recombined with the RetroQ plasmid digested with BamHI and EcoRI to form the ReteoQ-shRNA plasmids. PCRamplified sequences from $P T C 1$ (forward: 5'-tgctaaactacaaactggaa- 3 '; reverse: $5^{\prime}$-gctcattgattggaatttcc- $3^{\prime}$ ) and PTC2 (forward: $5^{\prime}$-gaccctccttaacgaattgt- $3^{\prime}$; reverse: $5^{\prime}$-gctccttgctgggcatctct- $3^{\prime}$ ) were recombined with BamHI and EcoRI digested at pEGFP-N1 to construct the pEGFP-PTC plasmids. The ReteoQ-shRNA and pEGFP-PTC plasmids were transformed, filtrated, amplified and co-transfected. Thirdgeneration lentiviral particles containing effective siRNAs were generated using the ReteoQ-shRNA packaging plasmid transfected with polyethyleneimine and stored as frozen stocks. Viral titers were determined using serial dilutions. Plasmids were screened for silencing via co-transfection with the GFP reporters. Seventy-two hours after co-transfection of ReteoQ-shRNA and pEGFP-PTC plasmids, fluorescence generated from the ReteoQ-shRNA plasmids was observed. The establishment of ReteoQ-shRNA plasmids was deemed successful if no green fluorescence was observed. Rat dorsal root ganglion (DRG) cells were cultured for 5 days, after which the cells were infected with lentivirus, cultured for another $48 \mathrm{~h}$ and subsequently lysed. Quantitative real-time polymerase chain reaction (qRT-PCR) and western blotting were performed to detect the expression of PTC mRNAs and proteins to evaluate viral interference.

\section{Experimental groups and viral intervention}

SCI rat models were assigned to the following seven groups: (1) normal group (healthy rats); (2) sham group (rats that had undergone anesthetization, neural plate fracture and exposure of the spinal cord without any injury treatment); (3) SCI group; (4) negative control (NC) group (SCI rats that received a spinal cord injection of $8 \mu \mathrm{l}$ lentivirus $\left(10^{7} \mathrm{TU} \mathrm{ml}^{-1}\right)$ containing ineffective shRNA); (5) PTC1RNAi group (SCI rats that received a spinal cord injection of $8 \mu \mathrm{l}$ of lentivirus ( $10^{7} \mathrm{TU} \mathrm{ml}^{-1}$ ) with PTC1-shRNA); (6) PTC2-RNAi group (SCI rats that received a spinal cord injection of $8 \mu \mathrm{l}$ of lentivirus $\left(10^{7} \mathrm{TU} \mathrm{ml}^{-1}\right)$ with PTC2-shRNA) and (7) PTC1/PTC2-RNAi group (SCI rats that received a spinal cord injection of $8 \mu \mathrm{l}$ of lentivirus $\left(10^{7} \mathrm{TU} \mathrm{ml}^{-1}\right)$ with PTC1/PTC2-shRNA). After the models were successfully established, the rats underwent thorough hemostasis and suturing of their respective incisions. Within 1-3 days after the operation, rats in the NC group were injected with antibiotics to help 
fight infection. Rat bladders were pressed gently two to three times daily until voluntary urination was obtained.

\section{Evaluation of hind limb motor function}

The hind limb motor function of the rats was evaluated using the Basso, Beattie and Bresnahan (BBB) scale on the 1st, 7th, 14th, 21st and 28th day after SCI. ${ }^{15}$ Rats were placed in a $125 \mathrm{~cm} \times 125 \mathrm{~cm}$ open space and were allowed to move freely. Hind limb motor function and body control in addition to all other relevant indicators were evaluated via attraction to stimuli for $4 \mathrm{~min}$ per evaluation. The 21-point BBB scale was used to grade the hind limb motor function of rats. In general, 0 points indicate complete paralysis and 21 points indicate normal locomotion. Scores ranging between 1 and 20 indicate a corresponding level of performance of the rat hind limbs. The detailed and basic scoring parameters were in accordance with a previous study, ${ }^{16}$ and a three-part evaluation was included. First, the degree of agility and other details relevant to the hind limb joint movements were evaluated; second, gait and coordination were assessed; third, fine movement of the rat toes was tallied.

\section{qRT-PCR}

After the evaluation of hind limb motor function, rat spinal cords were collected and digested followed by cell lysis. Total RNA was extracted using an RNA kit (Omega Bio-tek, Norcross, GA, USA). Reversetranscription was conducted in accordance with the instructions provided with the reverse transcription kit (Thermo Fisher Scientific, Sunnyvale, CA, USA). The cDNA was used as a template for PCR. Primer 5.0 software was employed to design primers, and $\beta$-actin was used as an internal reference. The primer sequences were as follows: Shh: forward: $5^{\prime}$-ccgaacgatttaaggaactcac- $3^{\prime}$, reverse: $5^{\prime}$-tgtctttgcacc tctgagtcat-3'; Gli-1: forward: $5^{\prime}$-ctctgctgactctgggatatg- $3^{\prime}$, reverse: $5^{\prime}$ gatcaggataggagcctgctg-3'; PTC1: forward: $5^{\prime}$-tgctaaactacaaactggaa-3', reverse: $5^{\prime}$-gctcattgattggaatttcc-3'; PTC2: forward: $5^{\prime}$-gaccctccttaacga attgt-3', reverse: $5^{\prime}$-gctccttgctgggcatctct- $3^{\prime}$; Nestin: forward: GCGGGG CGGTGCGTGACTAC, reverse: AGGCAAGGGGGAAGAGAAGG ATGT; Smo: forward: GCAGTTCCTCGGCTGCCTC, reverse: AGCC TCCATTAGGTTAGTGCG; and $\beta$-actin: forward: $5^{\prime}$-tgggacgacatg gagaaaa- $3^{\prime}$, reverse: $5^{\prime}$-ctggaaggtggacagcgag- $3^{\prime}$. The threshold cycle $(\mathrm{Ct})$ values were determined and used to quantify the relative levels of PTC1, PTC2, Gli-1 and Shh mRNAs using the $2^{-\Delta \Delta \mathrm{Ct}}$ method.

\section{Western blotting}

The collected $100 \mathrm{mg}$ of spinal cord tissue from rats in all 7 groups was washed with phosphate-buffered saline (PBS). The total protein amount was extracted according to the procedural instructions provided by the tissue protein extraction kit (No. P060091; Shanghai Andi Biotechnology Co., Ltd, Shanghai, China) and separated by sodium dodecyl sulfate polyacrylamide gel electrophoresis (SDSPAGE). Next, the total protein was electrically transferred onto a polyvinylidene fluoride (PVDF) membrane for detection. Antibodies were used at the following dilution ratios: rat anti-PTC1 (Santa Cruz; SC-6147; 1: 1000), rat anti-PTC2 (Proteintech; 55091-1-AP; 1: 1000), rat anti-Shh (Santa Cruz; SC-365112; 1: 1000), rat anti-Gli-1 (Santa Cruz; SC-515751; 1 : 1000$)$ and rat anti- $\beta$-actin (Santa Cruz; SC-7210; 1: 800) served as an internal reference. Membranes were incubated overnight at $4{ }^{\circ} \mathrm{C}$ and then added to horseradish peroxidase (HRP)marked secondary antibodies (1: 200; \#3999; cell signaling) for $2 \mathrm{~h}$ at room temperature. The gel imaging system (Gel Doc XR+, Bio-Rad Laboratories, Inc., Hercules, CA, USA) was used to obtain photographs, and ImageJ software was used to test gray levels.

\section{Hematoxylin and eosin staining}

On the 28th day following motor function evaluation, the rats were killed, and spinal cord tissues were dissected and processed for preparation of paraffin-embedded sections. The tissues were fixed in $4 \%$ methyl alcohol solution, paraffin-embedded, sectioned and dewaxed. Sections were washed with PBS and stained with hematoxylin and eosin (HE) as follows. First, sections were fixed with acetone for $2 \mathrm{~min}$, washed with water for 1-2 s, and then stained with hematoxylin for $5 \mathrm{~min}$ at room temperature. The sections were then washed with water for 5-10 min, immersed in 1\% acidic alcohol for $5-10 \mathrm{~s}$ and rinsed twice ( $2 \mathrm{~min}$ per rinse) with distilled water. Eosin solution was added to re-stain the sections for $1-2 \mathrm{~min}$. The sections were then washed with distilled water for $1-2 \mathrm{~s}$ and dehydrated using a gradient ethanol series $(80 \%, 90 \%$ and $100 \% ; 30$ s per step). Finally, the sections were immersed in xylene I ( $5 \mathrm{~min}$ ) and xylene II (5 min) and sealed with neutral gum. Stained tissues were observed under an optical microscope.

\section{Immunohistochemistry}

On the 28th day after motor function evaluation, the rats were killed, and spinal cord tissues were dissected and processed to prepare paraffin-embedded sections. The tissues were fixed in a $4 \%$ methyl alcohol solution, paraffin-embedded, sectioned, dewaxed and washed with PBS. The dewaxed and hydrated sections were placed in boiling buffer (citrate buffer; $\mathrm{pH}=6.0$ ) for $10 \mathrm{~min}$. Each section was then incubated in one drop of $3 \% \mathrm{H}_{2} \mathrm{O}_{2}$ for $10 \mathrm{~min}$ and in one drop of primary monoclonal antibodies (Neurofilament-200; NF-200; 1: 500 or glial fibrillary acidic protein; GFAP; 1: 200; Fuzhou Maixin Biotech., Fujian, China) for $2 \mathrm{~h}$. The sections were washed with PBS, incubated with avidin-conjugated secondary antibodies (1: 800; Fuzhou Maixin Biotech.) for $20 \mathrm{~min}$, washed again with PBS and incubated with a drop of HRP-marked rabbit anti-mouse polymer for $30 \mathrm{~min}$. Freshly prepared diaminobenzidine (3, $3^{\prime}$-diaminobenzidine, DAB) solution was added to each section (one drop), and the staining process was monitored for 5 min under a microscope. Sections were then counterstained, differentiated and blued. Finally, the sections were dehydrated, dried, sealed, aired and photographed. The criteria for scoring were based on positive astrocytes and neurofilaments (previously described). ${ }^{17}$ When 50 high-power fields were counted, the percentage of positive cells of 1000 cells was used to indicate the level of expression, with $<50 \%$ denoted as $(-)$ and $\geqslant 50 \%$ as $(+)$.

\section{Statistical analysis}

SPSS 19.0 software (SPSS, Chicago, IL, USA) was employed for statistical analysis. Measurement data are displayed as the mean \pm s.d. Comparisons between two groups were performed using the Independent Sample's t-test, while comparisons among multiple groups were conducted by one-way analysis of variance (ANOVA). Multiple comparisons of means were performed using the least significant difference method when there were no differences in the variances or by the Games-Howell method when there were differences in the variances. In addition, the cell-staining data are presented as percentages/ratios. The Chi-square test was also conducted for comparative analysis. $P<0.05$ indicated statistical significance.

\section{RESULTS}

\section{Successful construction of siRNA plasmids}

The fluorescence microscopy results revealed green fluorescence from the pEGFP-PTC1, pEGFP-PTC2 and pEGFP- 
PTC1/2 plasmids (Figure 1). Seventy-two hours after cotransfection of ReteoQ-shRNA-1-PTC1, ReteoQ-shRNA-1PTC2 or ReteoQ-shRNA-1-PTC1/2 and pEGFP-PTC, no green fluorescence was observed, which indicated that the PTC-RNAi plasmids had suppressed expression of the complementary pEGFP-PTC reporters. This result indicated the successful construction of the siRNA-1-PTC1, siRNA-1-PTC2 and siRNA-1-PTC1/2 plasmids. By contrast, $72 \mathrm{~h}$ after cotransfection of ReteoQ-shRNA-2-PTC1, ReteoQ-shRNA-2PTC2 or ReteoQ-shRNA-2-PTC1/2 and pEGFP-PTC, green fluorescence was still visible, indicating that the siRNA-2PTC1, siRNA-2-PTC2 and siRNA-2-PTC1/2 plasmids had not been successfully constructed. Lentiviral particles in the third generation containing effective ReteoQ-shRNA-1-PTC1, ReteoQ-shRNA-1-PTC2 and ReteoQ-shRNA-1-PTC1/2 plasmids were obtained using the polyethyleneimine method and were used for subsequent experiments.

mRNA and protein expression of PTC1 and PTC2 in DRG cells infected with lentivirus

Two days after DRG cells were infected with RNAi lentivirus, the levels of PTC1 and PTC2 mRNA were measured, as shown in Figure 2. The PTC1-RNAi and PTC1/2-RNAi groups exhibited significantly lower PTC1 mRNA levels than the control group (both $P<0.05$ ). The PTC2-RNAi and PTC1/2RNAi groups showed significantly lower mRNA levels of PTC2 than the control group (both $P<0.05$ ). The protein levels of PTC1 and PTC2 were determined 2 days after DRG cells were infected with lentivirus, as depicted in Figure 3. Compared with the control group, the PTC1-RNAi and PTC1/2-RNAi groups exhibited a marked decrease in PTC1 protein levels (both


Figure 1 Representations of images of cells transfected with PTC1-RNAi, PTC2-RNAi and PTC1/PTC2-RNAi plasmids using fluorescence microscopy $(\times 200)$. Notes: (a) cells transfected with the pEGFP-PTC1 plasmid; (b) cells transfected with the pEGFP-PTC2 plasmid; (c) cells transfected with the pEGFP-PTC1/2 plasmid; (d) cells co-transfected with pEGFP-PTC1+ReteoQ-shRNA-1-PTC1 plasmids; (e) cells co-transfected with pEGFP-PTC2+ReteoQ-shRNA-1-PTC2 plasmids; (f) cells co-transfected with pEGFP-PTC1/2+ReteoQ-shRNA-1-PTC1/2 plasmids; (g) cells co-transfected with pEGFP-PTC1+ReteoQ-shRNA-2-PTC1; (h) cells co-transfected with pEGFP-PTC2+ReteoQ-shRNA-2PTC2 plasmids; (i) cells co-transfected with pEGFP-PTC1/2+ReteoQ-shRNA-2-PTC1/2 plasmids; EGFP, enhanced green fluorescent protein; PTC1, Patched 1; PTC2, Patched 2. 
$P<0.05$ ), and the PTC2-RNAi and PTC1/2-RNAi groups displayed significantly lower PTC2 protein levels (both $P<0.05)$. The above results supported successful lentivirusmediated shRNA interference.

\section{Comparison of BBB scores of rats in the seven groups at different time points}

Prior to SCI, all the selected rats in the study had 21 points based on the BBB scales. After SCI, all the rats exhibited paralyzed hind legs, tail dysfunctions, no responses to acupuncture, urination disorders and reduced diets (all $P<0.05$ ). As time progressed, the BBB scores of the SCI rats gradually increased. Seven days after injury, SCI rats could move one joint at most. Fourteen days after injury, SCI rats in the PTC1RNAi, PTC2-RNAi and PTC1/PTC2-RNAi groups exhibited significantly increased ranges of hind limb joint motion, while those in the NC and SCI groups typically showed only mild joint motion. Twenty-one days after injury, rats in the PTC1RNAi, PTC2-RNAi and PTC1/PTC2-RNAi groups were capable of dragging a weighted load and exhibited improved urination function. Twenty-eight days after injury, rats in the PTC1/PTC2-RNAi group placed their hind feet flat on the floor and received 9 points on the BBB scales. The recovery of rats in the PTC1/PTC2-RNAi group was significantly better than that in the NC, SCI, PTC1-RNAi and PTC2-RNAi groups (all $P<0.05)$. The subsequent recovery of rats in the PTC1-RNAi and PTC2-RNAi groups was superior to that in the NC and SCI groups (all $P<0.05$ ). In relation to the BBB scales, rats in the NC and SCI groups had lower scores than those in the normal, sham, PTC1-RNAi, PTC2-RNAi and PTC1/PTC2RNAi groups. No significant difference in $\mathrm{BBB}$ scores was observed between the $\mathrm{NC}$ and SCI groups (all $P>0.05$ ) (Table 1).

\section{Comparison of Shh, Gli-1, PTC1 and PTC2 mRNA expression among the seven groups}

qRT-PCR revealed evidence concerning the expression of Shh, Gli-1 and Smo in all seven groups. Rats in the SCI, NC, PTC1RNAi, PTC2-RNAi, and PTC1/PTC2-RNAi groups showed elevated expression levels of Shh, Gli-1 and Smo, which peaked on the 7th day and remained high until the 28th day $(P<0.05)$. Rats in the PTC1/PTC2-RNAi group showed the highest mRNA levels of Shh, Gli-1 and Smo among all seven groups (all $P<0.05$ ). In addition, the Shh, Gli-1 and Smo mRNA levels were significantly elevated in the PTC1-RNAi and PTC2RNAi groups compared with the SCI and normal groups
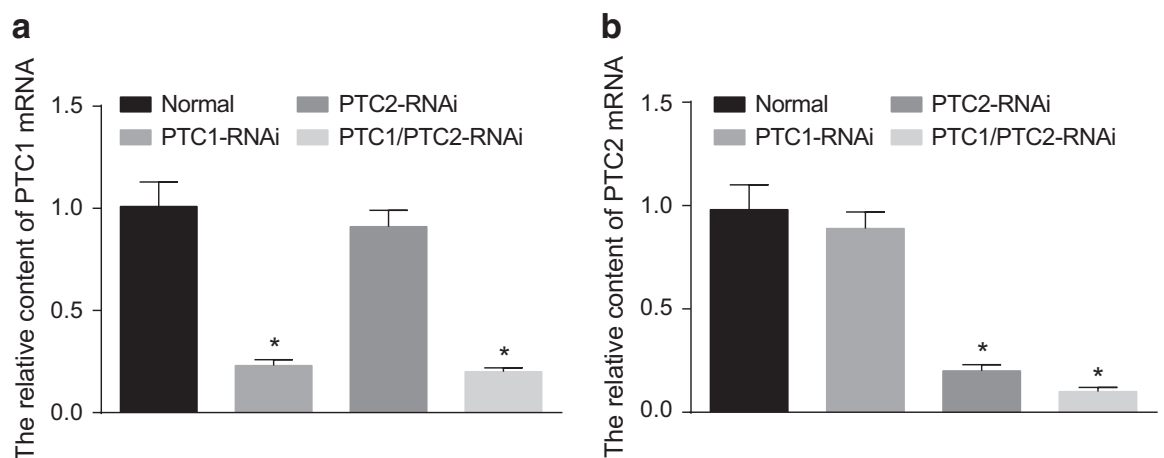

Figure 2 Comparisons of PTC1 and PTC2 mRNA expression in DRG cells among the normal, PTC1-RNAi, PTC2-RNAi and PTC1/PTC2RNAi groups. Notes: (a) relative PTC1 mRNA expression in DRG cells among the four groups; (b) relative PTC2 mRNA expression in DRG cells among the four groups; PTC1, Patched 1; PTC2, Patched 2; DRG, dorsal root ganglion; ${ }^{*} P<0.05$ compared with the normal group.
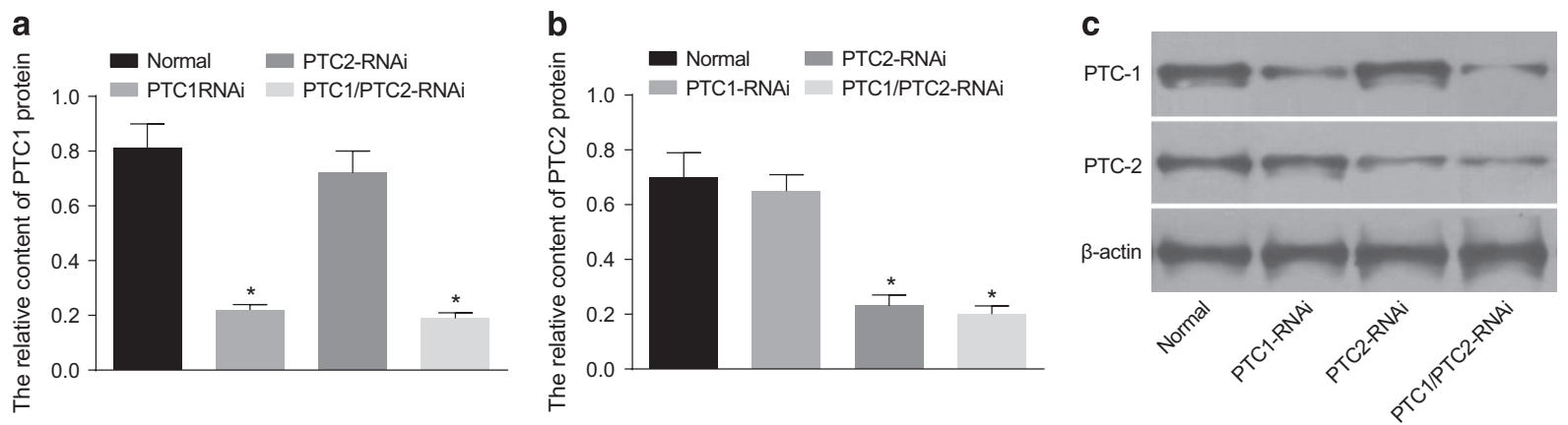

Figure 3 Comparisons of PTC1 and PTC2 protein expression in DRG cells among the normal, PTC1-RNAi, PTC2-RNAi and PTC1/PTC2RNAi groups. Notes: (a) relative PTC1 protein expression in DRG cells among the four groups; (b) relative PTC2 protein expression in DRG cells among the four groups; (c) relative gray values of PTC1 and PTC2 proteins detected by western blotting in the four groups; PTC1, Patched 1; PTC2, Patched 2; DRG, dorsal root ganglion; RNAi, RNA interference; ${ }^{*} P<0.05$ compared with the normal group. 
$(P<0.05)$; however, no apparent difference was observed between the PTC1-RNAi and the PTC2-RNAi groups (all $P>0.05)$. Compared with the normal group, the SCI group exhibited significantly increased Shh, Gli-1 and Smo mRNA levels $(P<0.05)$. Moreover, the mRNA levels of Nestin, despite an obvious increase after SCI, were relatively elevated in the PTC1-RNAi, PTC2-RNAi and PTC1/PTC2-RNAi groups. Comparatively, the PTC1/PTC2-RNAi group exhibited the greatest increase in this regard $(P<0.05)$ (Figure 4$)$.

The PTC1-RNAi and PTC1/PTC2-RNAi groups showed significant decreases in mRNA expression of PTC1, in parallel to the normal, sham, SCI, NC and PTC2-RNAi groups $(P<0.05)$. In addition, the PTC2-RNAi and PTC1/PTC2-RNAi groups also displayed rather apparent decreases in mRNA expression of PTC2 in comparison to the other five groups $(P<0.05)$ (Figure 4$)$. The SCI and NC groups exhibited the highest mRNA expression of PTC1 and PTC2, which gradually decreased as time progressed but remained significantly higher than that in the normal group $(P<0.05)$.

\section{Comparison of Shh, Gli-1, PTC1 and PTC2 protein expression among the seven groups}

Figure 5 illustrates that the Shh, Gli-1, PTC1, PTC2, Smo and Nestin proteins were expressed in all seven groups. Following the observed increase in Shh, Gli-1, Smo and Nestin protein in the SCI rats, lower protein levels of PTC1 and PTC2 were detected. Western blotting analysis revealed the highest levels of Shh, Gli-1, Smo and Nestin protein in the PTC1/PTC2-RNAi group, in sharp contrast to the protein levels observed in the PTC1-RNAi, PTC2-RNAi, normal and SCI groups (all $P<0.05)$. The PTC1-RNAi and PTC2-RNAi groups exhibited notably higher Shh, Gli-1, Smo and Nestin protein expressions than did the normal and SCI groups (all $P<0.05$ ). Moreover, the PTC1-RNAi and PTC1/PTC2-RNAi groups displayed a marked decrease in PTC1 protein expression levels compared with the normal, sham, SCI, NC and PTC2-RNAi groups (all $P<0.05)$. Significantly decreased expression of PTC2 protein was observed in the PTC2-RNAi and PTC1/PTC2-RNAi groups compared with the normal, sham, SCI, NC and PTC1-RNAi groups (all $P<0.05$ ).
Histomorphology of HE-stained spinal tissue from the seven groups

Twenty-eight days after SCI, HE staining revealed that rats in the SCI and NC groups exhibited degeneration, necrosis of neurocytes, as well as necrosis in cystic cavities. These rats also exhibited loose and disordered nerve fibers within the substantia alba. However, in the PTC1-RNAi, PTC2-RNAi and PTC1/PTC2-RNAi groups, the rats had less inflammation, as well as spinal cords that were regularly structured with no obvious cavities. In addition, certain areas exhibited rather noticeable nerve regeneration, as well as displays of complete structures (Figure 6).

\section{Comparison of NF-200-positive areas among the seven groups}

Twenty-eight days after SCI, immunohistochemistry in NF-200 revealed that the PTC1-RNAi, PTC2-RNAi and PTC1/PTC2RNAi groups exhibited increased numbers of cells with relatively larger bodies accompanied by obvious nerve regeneration. Image-Pro Plus 6.0 software was used to calculate and analyze the NF-200-positive areas. The obtained results are represented in Figure 7. The PTC1/PTC2-RNAi group showed a significantly larger NF-200-positive area than the normal, sham, SCI, NC, PTC1-RNAi and PTC2-RNAi groups (all $P<0.05)$. Compared with the SCI, NC and normal groups, the PTC1-RNAi and PTC2-RNAi groups also exhibited significantly larger NF-200-positive areas (all $P<0.05$ ). The SCI group showed a significantly larger NF-200-positive area than the normal group $(P<0.05)$. Regarding the statistical analysis, there were no significant differences in this regard between the PTC1-RNAi and PTC2-RNAi groups (all P>0.05) (Figure 7).

Comparison of GFAP-positive areas among the seven groups Figure 8 shows that astrocytes in the spinal cords of the rats in all seven groups had similar expression levels of GFAP. ImagePro Plus 6.0 software was employed to calculate and analyze the positive areas. The SCI, NC, PTC1-RNAi, PTC2-RNAi and PTC1/PTC2-RNAi groups all had significantly larger GFAPpositive areas than the normal and sham groups (all $P<0.05$ ). No significant differences in GFAP-positive areas were detected among the PTC1-RNAi, PTC2-RNAi, PTC1/PTC2-RNAi groups and the SCI, NC groups (all $P>0.05$ ).

Table 1 Comparison of BBB scores before and after operation among the normal, sham, SCI, NC, PTC1-RNAi, PTC2-RNAi and PTC1/PTC2-RNAi groups

\begin{tabular}{lccccccc}
\hline BBB scale & Normal group & Sham group & SCI group & NC group & PTC1-RNAi group & PTC2-RNAi group & PTCI/PTC2-RNAi group \\
\hline 1 d before operation & $21 \pm 0.0$ & $21 \pm 0.0$ & $21 \pm 0.0$ & $21 \pm 0.0$ & $21 \pm 0.0$ & $21 \pm 0.0$ & $21 \pm 0.0$ \\
1 d after operation & $21 \pm 0.0$ & $20.4 \pm 0.3$ & $0.1 \pm 0.1^{*}$ & $0.1 \pm 0.1^{*}$ & $0.1 \pm 0.1^{*}$ & $0.2^{*} \pm .2^{*}$ & $0.18 \pm 0.1^{*}$ \\
7 d after operation & $21 \pm 0.0$ & $20.5 \pm 0.4$ & $1.74 \pm 0.8^{*}$ & $1.82 \pm 0.6^{*}$ & $1.9 \pm 0.7^{*}$ & $1.9 \pm 0.6^{*}$ & $2.1 \pm 0.4^{*}$ \\
14 d after operation & $21 \pm 0.0$ & $20.6 \pm 0.3$ & $2.7 \pm 1.2^{*}$ & $2.8 \pm 0.7^{*}$ & $4.8 \pm 1.5^{*}, \#$ & $4.9 \pm 1.6^{*}, \#$ & $5.8 \pm 1.6^{*}, \#$ \\
21 d after operation & $21 \pm 0.0$ & $20.8 \pm 0.3$ & $3.7 \pm 1.8^{*}$ & $3.75 \pm 1.7^{*}$ & $7.1 \pm 0.9^{*}, \#$ & $7.5 \pm 1.8^{*}, \#$ & $11.92 \pm 1.5^{*}, \#, \&$ \\
28 d after operation & $21 \pm 0.0$ & $20.9 \pm 0.2$ & $5.5 \pm 1.8^{*}$ & $5.4 \pm 1.9^{*}$ & $9.5 \pm 1.9^{*}, \#$ & $9.8 \pm 2.1^{*}, \#$ & $14.80 \pm 1.9^{*}, \#, \&$ \\
\hline
\end{tabular}

Abbreviations: BBB, Basso, Beattie and Bresnahan; d, day; NC, negative control; PTC1, Patched 1; PTC2, Patched 2; SCI, spinal cord injury.

$* P<0.05$ in comparison with the normal group.

${ }^{\#} P<0.05$ in comparison with the $\mathrm{SCl}$ and $\mathrm{NC}$ groups.

$\& P<0.05$ in comparison with the PTC1-RNAi and PTC2-RNAi groups. 


\section{DISCUSSION}

Due to an increasing annual incidence, SCI remains highly prevalent worldwide. ${ }^{18}$ Recovery from SCI is difficult to achieve due to the inability of severed axons of the central nervous system to spontaneously regenerate. ${ }^{19}$ Key inhibitory obstacles that impede axonal regeneration include the glial scar and a number of myelin inhibitory molecules. ${ }^{20}$ Thus, there is an neural regeneration.

The qRT-PCR and western blotting techniques employed demonstrated an increase in the expression of PTC1 and PTC2 after SCI. Rats in the PTC1/PTC2-RNAi group exhibited higher Shh and Gli-1 mRNA and protein expression levels. However, the rats in the aforementioned group displayed lower PTC1 and PTC2 mRNA and protein expression levels than the

urgent need for progressive therapeutic strategies to promote

NC, SCI, PTC1-RNAi and PTC2-RNAi groups. This observation suggested that PTC1 and PTC2 gene silencing activated the Hh signaling pathway. Previous studies have indicated that the transcription of Glil in the nervous system and limbs is dependent on the Shh pathway. ${ }^{21}$ A prior study has also reported that when Glil is absent in nervous tissue, enteric neurons do not respond to $\mathrm{Hh}$ signals that can upregulate Ptch1 expression. ${ }^{22}$ Schnapp et al. ${ }^{23}$ reported that PTC1 is one of the target genes of the Hh signaling pathway, which is upregulated by Shh signaling. PTC2 has been shown to modulate tumorigenesis with PTC1 haplo-insufficiency. Furthermore, medulloblastomas that develop from perturbations of PTC1 function exhibit a concomitant up-regulation of PTC2. ${ }^{24}$ In addition, germline inactivation of Ptch1 has been reported to be a predisposing factor in basal cell carcinoma in
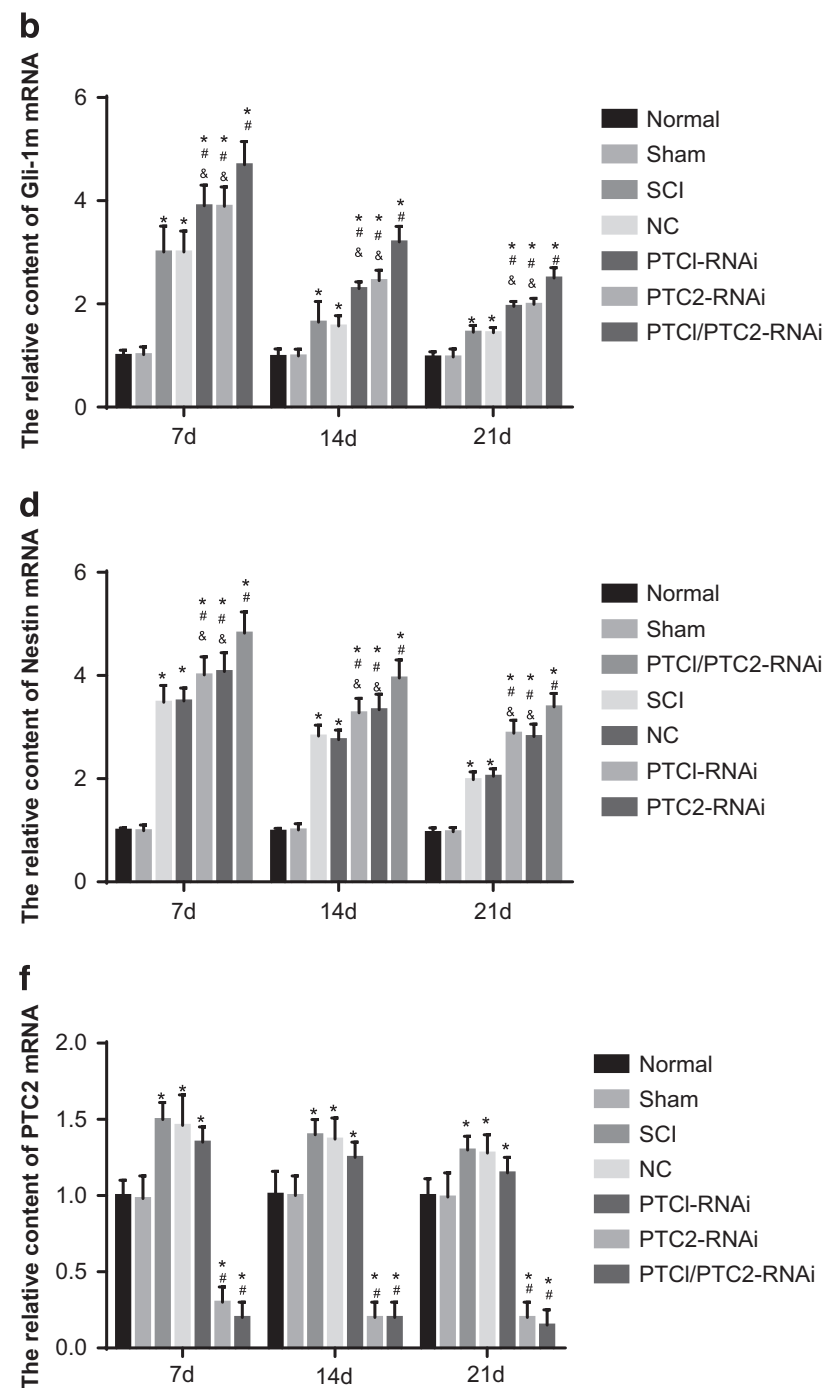

Figure 4 Comparison of Shh, Gli-1, PTC1, PTC2, Nestin and Smo mRNA expression among the seven groups. Notes: (a) relative Shh mRNA expression among the seven groups; (b) relative Gli-1 mRNA expression among the seven groups; (c) relative Smo mRNA expression among the seven groups; (d) relative Nestin mRNA expression among the seven groups; (e) relative PTC1 mRNA expression among the seven groups; (f) relative PTC2 mRNA expression among the seven groups; Shh, sonic hedgehog; Gli-1, glioma-associated oncogene homolog 1; PTC1, Patched 1; PTC2, Patched 2; SCI, spinal cord injury; NC, negative control; * $P<0.05$ compared with the normal group; $\# P<0.05$ compared with the SCl group; \& $P<0.05$ compared with the PTC1/PTC2-RNAi group. 
a


f
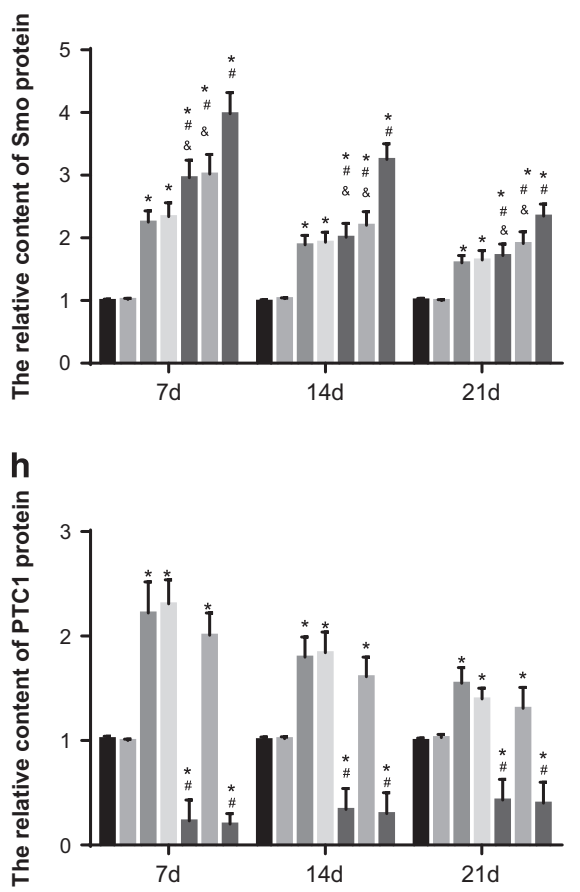

b

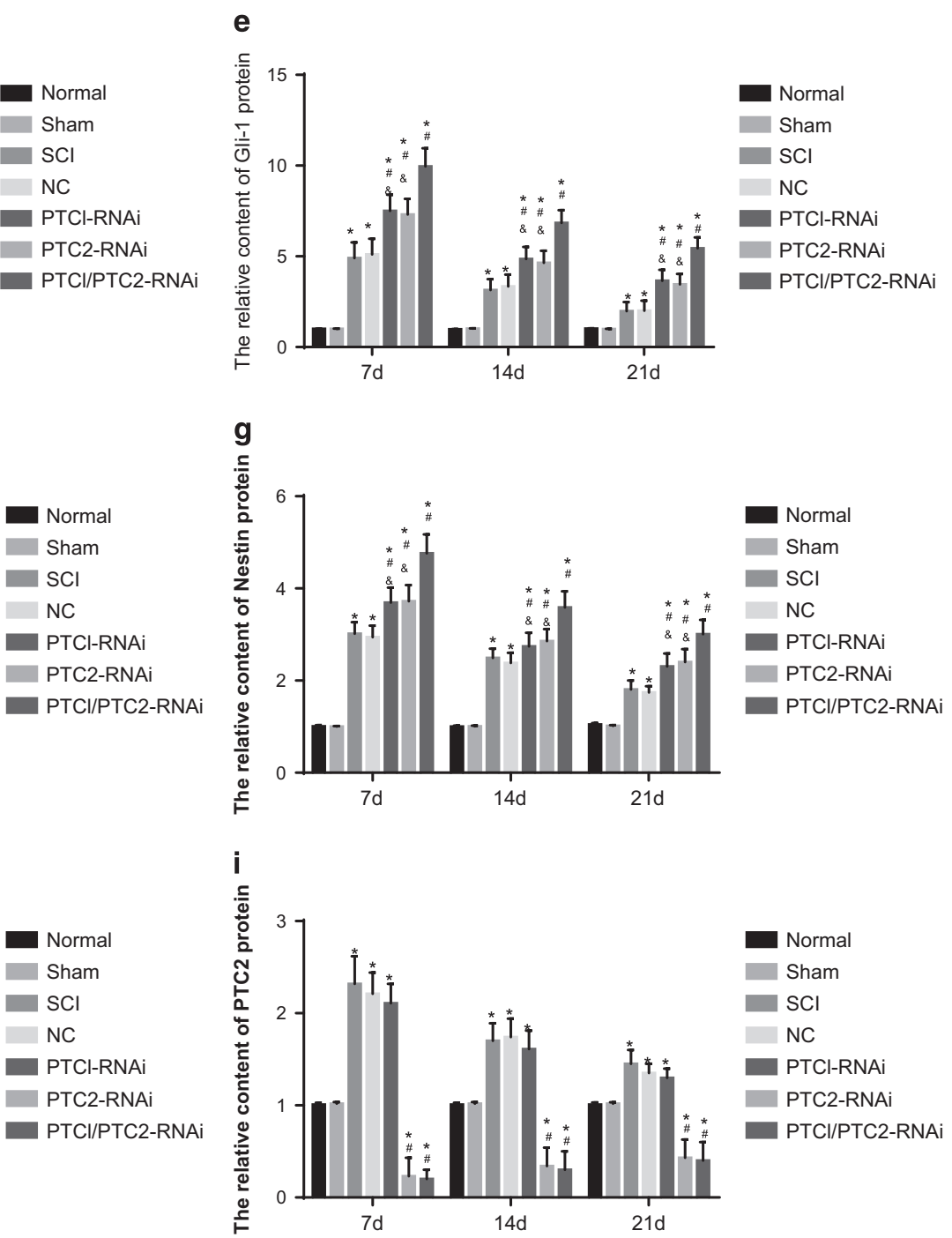

Figure 5 Comparison of the relative expression of PTC1, PTC2, Shh, Gli-1, Nestin and Smo proteins among the seven groups on the 7th, 14th and 28th days. Notes: (a) relative expression of PTC1, PTC2, Shh, Gli-1, Nestin and Smo proteins on the 7th day; (b) relative expression of PTC1, PTC2, Shh, Gli-1, Nestin and Smo proteins on the 14th day; (c) relative expression of PTC1, PTC2, Shh, Gli-1, Nestin and Smo proteins on the 21st day; (d) relative expression of Shh protein; (e) relative expression of Gli-1 protein; (f) relative expression of Smo protein. Relative expression of Nestin protein; (h) relative expression of PTC1 protein; (i) relative expression of PTC2 protein; Gli-1, glioma-associated oncogene homolog 1; NC, negative control; PTC1, Patched 1; PTC2, Patched 2; SCl, spinal cord injury; Shh, sonic hedgehog; ${ }^{*} P<0.05$ compared with the normal group; ${ }^{\#} P<0.05$ compared with the SCI group; \& $P<0.05$ compared with the PTC1/PTC2RNAi group. 
both humans and mice. During this study, silencing of PTC1 and PTC2 was accompanied by the upregulation of Shh and Gli1. This phenomenon was particularly indicative of activation of the Hh signaling pathway, which may contribute to SCI recovery. A previous report has indicated that modulating the neuro-proliferative effects of the Shh signaling cascade plays a potential role in improving the effects of CNS injury, particularly in animal models of SCI. ${ }^{25}$ In mature mammalian tissues, the presence of cells with progenitor function is required for regeneration after injury. The Hh pathway has been reported to expand stem cell and progenitor cell numbers in various adult tissues. $^{26-28}$ Ochoa et al. ${ }^{29}$ reported that the activation of $\mathrm{Hh}$ signaling stimulates fibrogenic repair during liver injury and this activity correlates with the expansion of populations of myofibroblastic cells needed for liver cell regeneration. Moreover, studies have indicated that Shh signaling is able to induce endogenous neural precursor cells and neural stem cells, both of which are essential for neurodevelopment. ${ }^{25,30}$ The data collected during the study provided sufficient evidence allowing us to deduce that the Hh pathway is an important factor in regeneration after injury. Similarly, in the present study, PTC1 and PTC2 gene silencing elicited a downregulation effect on the expression of PTC1 and PTC2, while upregulating Shh and Gli, thus activating the Hh signaling pathway as well as promoting neural regeneration.

The BBB scale is the most frequently used method to evaluate functional recovery in rats after SCI. ${ }^{31}$ Twenty-eight days after SCI, rats in the PTC1/PTC2-RNAi group achieved higher BBB scores than those in the NC, SCI, PTC1-RNAi and PTC2-RNAi groups. Furthermore, the rats in the PTC1-RNAi and PTC2-RNAi groups had higher scores than those in the SCI and NC groups. The higher BBB scores in the PTC1/ PTC2-RNAi group suggested the greatest efficacy in regards to silencing PTC1 and PTC2, which asserted that silencing of

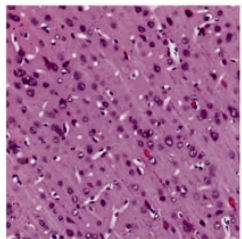

Normal

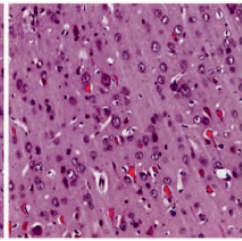

Sham

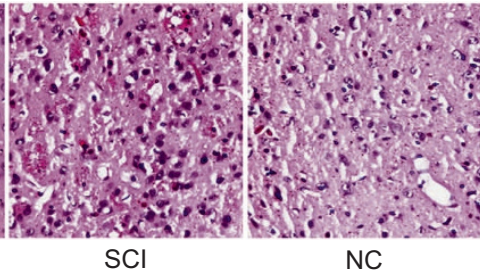

$\mathrm{NC}$

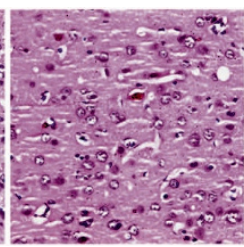

PTC1-RNAi

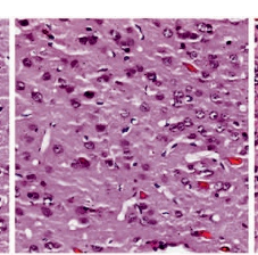

PTC2-RNAi



PTC1/2-RNAi

Figure 6 Histomorphology of HE-stained spinal tissues in the seven groups. Notes: Gli-1, glioma-associated oncogene homolog 1; HE, hematoxylin and eosin; NC, negative control; PTC1, Patched 1; PTC2, Patched 2; SCI, spinal cord injury; Shh, sonic hedgehog.
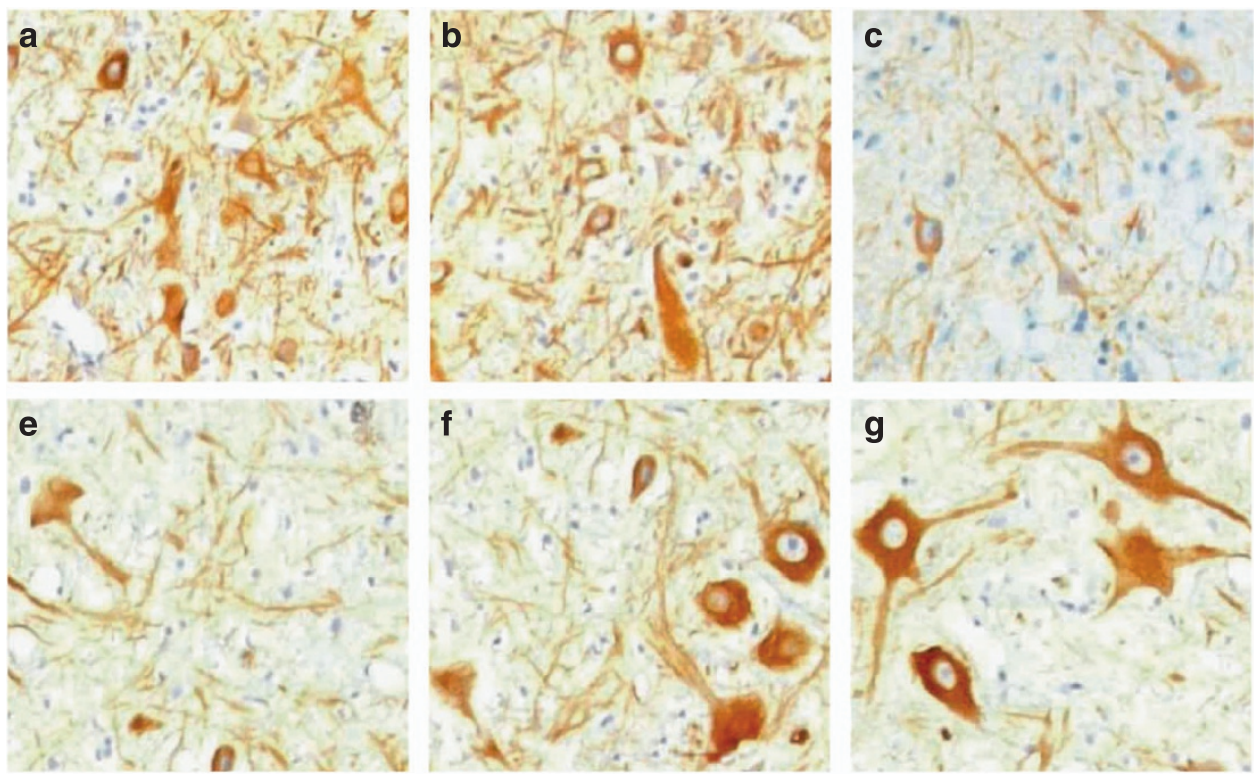



h



Figure 7 Comparison of the NF-200-positive areas detected by IHC among the seven groups (x20). Notes: (a) NF-200-positive areas in the normal group; (b) NF-200-positive areas in the sham group; (c) NF-200-positive areas in the SCl group; (d) NF-200-positive areas in the NC group; (e) NF-200-positive areas in the PTC1-RNAi group; (f) NF-200-positive areas in PTC2-RNAi group; (g) NF-200-positive areas in the PTC1/PTC2-RNAi group; (h) comparison of the percentages of NF-200-positive areas among the seven groups detected by IHC; Gli-1, glioma-associated oncogene homolog 1; IHC, immunohistochemistry; NC, negative control; NF-200, neurofilaments-200; PTC1, Patched 1; PTC2, Patched 2; SCI, spinal cord injury; Shh, sonic hedgehog; ${ }^{*} P<0.05$ compared with the normal group; ${ }^{\#} P<0.05$ compared with the $\mathrm{SCl}$ group; \& $P<0.05$ compared with the PTC1/PTC2-RNAi group. 
these genes is required to promote recovery in SCI patients. Bone marrow-derived mesenchymal stem cells (BMSCs) are a promising form of stem cell with important functions in SCI repair. It has previously been reported that Shh participates in the cartilaginous differentiation of mesenchymal cells in the spine and can promote cartilage formation in BMSCs in vitro. ${ }^{32}$ Shh can promote neuronal and BMSC survival, inhibit activation of the astrocyte lineage, facilitate axonal growth and strengthen the delivery of neurotrophic factors in $\mathrm{BMSCs}^{33}$ Zhang et al. ${ }^{34}$ indicated that activation of the Shh/Glil pathway may induce oligodendrogenesis, which could ultimately contribute to nerve recovery. Straface et al. have reported that the inhibition of Shh impairs the up-regulation of prototypical angiogenic agents, including stromal-derived factor (SDF)lalpha and vascular endothelial growth factor (VEGF). This phenomenon also accounts for decreases in muscle blood flow while reducing capillary density post injury. Shh inhibition results in increased inflammation as well as poor recovery of motor function after injury. ${ }^{9}$ Shh is capable of inducing important transcription factors such as Gli-1, which affects Bcl-2 expression (an anti-apoptotic effector) downstream of the Shh signaling pathway. ${ }^{35}$ As one of the direct targets of Shh signaling, Bcl2 can be regulated by Gli-1, which when inhibited by curcumin causes reduced cell growth in vitro and in vivo. ${ }^{36}$ Based on the above studies, the importance of Shh and Gli during SCI recovery was subsequently deduced. Increased Shh and Gli-1 may promote nerve regeneration due to activation of the Hh signaling pathway, which ultimately contributes to SCI recovery.

In the present study, HE staining revealed clear nerve regeneration in addition to a reduction in inflammation in rats in the PTC1-RNAi, PTC2-RNAi and PTC1/PTC2-RNAi groups. In addition, rats in the PTC1-RNAi, PTC2-RNAi and PTC1/ PTC2-RNAi groups exhibited increases in areas occupied by cells that were positive for NF-200 or GFAP. The Hh ligand acts as an anti-inflammatory epithelial modulator in mesenchymal inflammatory milieus, and acute modulation of Hh signaling changes inflammatory pathways in the intestinal mesenchyme. ${ }^{37}$ Smoothened (Smo), is a type of 7-pass transmembrane protein in the Hh pathway that is transferred to cilia upon acceptance of a ligand..$^{38}$ Moreover, myofibroblasts are capable of secreting inflammatory mediators ${ }^{39}$ and are responsive to the $\mathrm{Hh}$ signaling pathway. ${ }^{22}$ The reduced expression of $\mathrm{Hh}$ signaling causes mislocalization of myofibroblasts during late gestation and at times in the postnatal period. ${ }^{37}$ It has been previously suggested that when Shh is bound to PTC1 it moves away from cilia, activating the $\mathrm{Hh}$ signaling pathway. ${ }^{40}$ Activation of the $\mathrm{Hh}$ signaling pathway by silencing PTC1 and PTC2 may reduce inflammation to promote recovery. Because NF-200 is a neural marker ${ }^{41}$ and both NF-200 and GFAP are expressed in differentiated neurospheres, ${ }^{42}$ the increase in positive areas of NF-200 and GFAP in the PTC1/PTC2-RNAi group supports the hypothesis that $P T C 1$ and $P T C 2$ gene silencing promotes nerve regeneration.
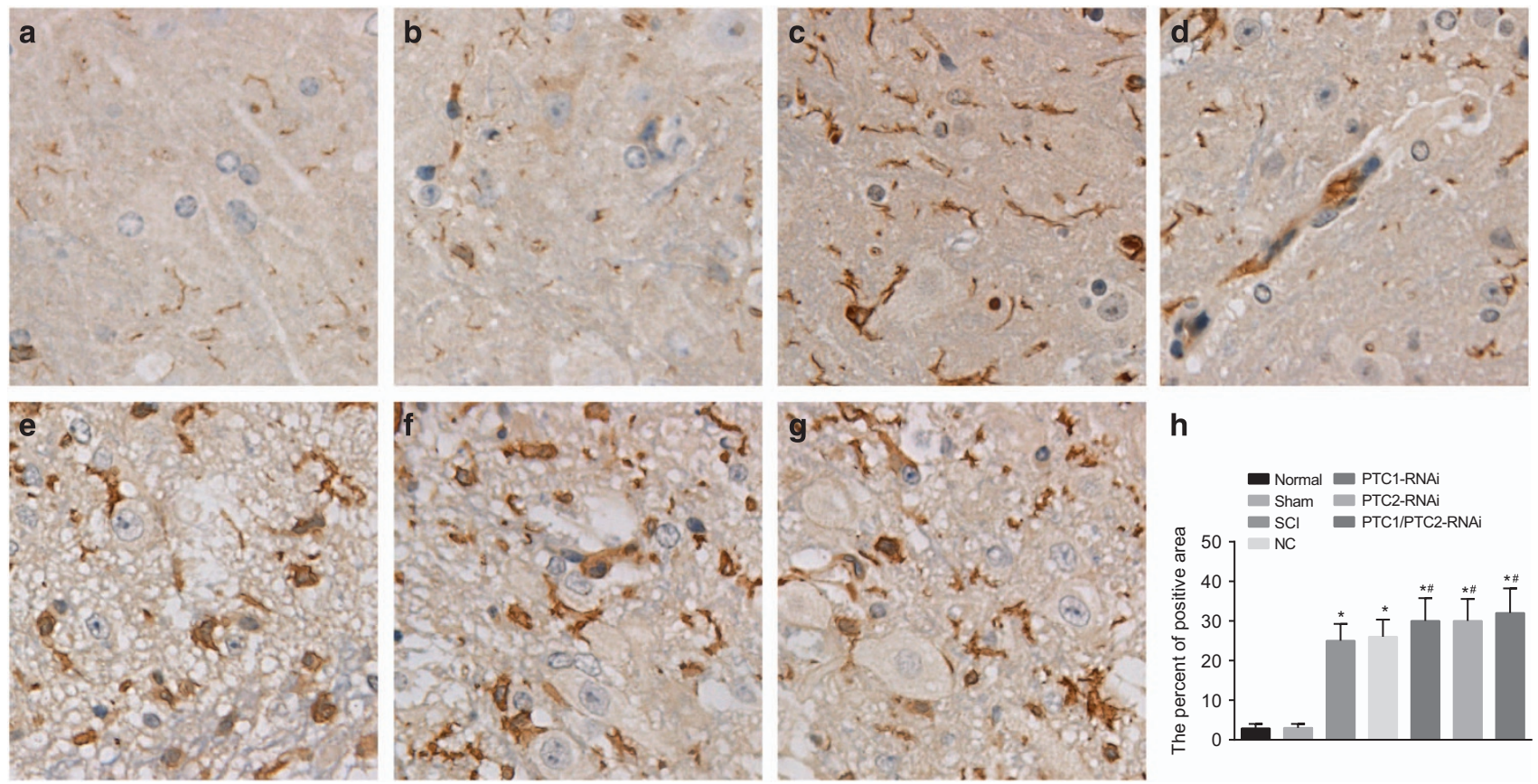

Figure 8 Comparison of the GFAP-positive areas detected by immunofluorescent staining among the seven groups. Notes: (a) GFAPpositive areas in the normal group; (b) GFAP-positive areas in the sham group; (c) GFAP-positive areas in the SCl group; (d) GFAP-positive areas in the NC group; (e) GFAP-positive areas in the PTC1-RNAi group; (f) GFAP-positive areas in the PTC2-RNAi group; (g) GFAPpositive areas in the PTC1/PTC2-RNAi group; (h) comparison of the percentages of GFAP-positive areas among the seven groups; GFAP, glial fibrillary acidic protein; Gli-1, glioma-associated oncogene homolog 1; NC, negative control; PTC1, Patched 1; PTC2, Patched 2; SCI, spinal cord injury; Shh, sonic hedgehog; ${ }^{*} P<0.05$ compared with the normal group; ${ }^{*} P<0.05$ compared with the $\mathrm{SCl}$ and $\mathrm{NC}$ group. 
In conclusion, the present study provides significant evidence that silencing of the PTC1 and PTC2 genes can accelerate the recovery of rats from SCI by activating the Hh signaling pathway. These findings further suggest that silencing of PTC1 and PTC2 is a promising strategy for accelerating recovery in patients with SCI. However, further research is required to explore the finer mechanistic details by which SCI promotes Shh and Gli-1 expression and activates the Hh signaling pathway.

\section{CONFLICT OF INTEREST}

The authors declare no conflict of interest.

\section{ACKNOWLEDGEMENTS}

This study was supported in part by National Natural Science Foundation of China (No.51672191 and 81601886). We would also like to express our sincere appreciation to the reviewers for critical comments on this manuscript.

\section{PUBLISHER'S NOTE}

Springer Nature remains neutral with regard to jurisdictional claims in published maps and institutional affiliations.

1 Domingo A, Al-Yahya AA, Asiri Y, Eng JJ, Lam T. Spinal cord injury rehabilitation evidence research. A systematic review of the effects of pharmacological agents on walking function in people with spinal cord injury. J Neurotrauma 2012; 29: 865-879.

2 Brambilla R, Bracchi-Ricard V, Hu WH, Frydel B, Bramwell A, Karmally S et al. Inhibition of astroglial nuclear factor kappaB reduces inflammation and improves functional recovery after spinal cord injury. J Exp Med 2005; 202: $145-156$.

3 Meletis K, Barnabe-Heider F, Carlen M, Evergren E, Tomilin N, Shupliakov O et al. Spinal cord injury reveals multilineage differentiation of ependymal cells. PLoS Biol 2008; 6: e182.

4 Thuret S, Moon LD, Gage FH. Therapeutic interventions after spinal cord injury. Nat Rev Neurosci 2006; 7: 628-643.

5 Hagg T, Oudega M. Degenerative and spontaneous regenerative processes after spinal cord injury. J Neurotrauma 2006; 23: 264-280.

6 Kusano KF, Allendoerfer KL, Munger W, Pola R, Bosch-Marce M, Kirchmair $\mathrm{R}$ et al. Sonic hedgehog induces arteriogenesis in diabetic vasa nervorum and restores function in diabetic neuropathy. Arterioscler Thromb Vasc Biol 2004; 24: 2102-2107.

7 Luo JD, Hu TP, Wang L, Chen MS, Liu SM, Chen AF. Sonic hedgehog improves delayed wound healing via enhancing cutaneous nitric oxide function in diabetes. Am J Physiol Endocrinol Metab 2009; 297: E525-E531.

8 Nakashima H, Nakamura M, Yamaguchi H, Yamanaka N, Akiyoshi T, Koga $\mathrm{K}$ et al. Nuclear factor-kappaB contributes to hedgehog signaling pathway activation through sonic hedgehog induction in pancreatic cancer. Cancer Res 2006; 66: 7041-7049.

9 Straface G, Aprahamian T, Flex A, Gaetani E, Biscetti F, Smith RC et al. Sonic hedgehog regulates angiogenesis and myogenesis during post-natal skeletal muscle regeneration. J Cell Mol Med 2009; 13: 2424-2435.

10 Edwards PC, Ruggiero S, Fantasia J, Burakoff R, Moorji SM, Paric E et al. Sonic hedgehog gene-enhanced tissue engineering for bone regeneration. Gene Ther 2005; 12: 75-86.

11 Hegde GV, Munger CM, Emanuel K, Joshi AD, Greiner TC, Weisenburger DD et al. Targeting of sonic hedgehog-GLI signaling: a potential strategy to improve therapy for mantle cell lymphoma. Mol Cancer Ther 2008; 7: $1450-1460$.

12 Li Z, Zhang H, Denhard LA, Liu LH, Zhou H, Lan ZJ. Reduced white fat mass in adult mice bearing a truncated Patched 1. Int J Biol Sci 2008; 4: 29-36.

13 Briscoe J, Therond P. Hedgehog signaling: from the Drosophila cuticle to anti-cancer drugs. Dev Cell 2005; 8: 143-151.
14 Duan C, Liu Y, Lu L, Cai R, Xue H, Mao X et al. CDK14 contributes to reactive gliosis via interaction with cyclin $Y$ in rat model of spinal cord injury. J Mol Neurosci 2015; 57: 571-579.

15 Zhou YJ, Liu JM, Wei SM, Zhang YH, Qu ZH, Chen SB. Propofol promotes spinal cord injury repair by bone marrow mesenchymal stem cell transplantation. Neural Regen Res 2015; 10: 1305-1311.

16 Costa LM, Pereira JE, Filipe VM, Magalhaes LG, Couto PA, Gonzalo-Orden JM et al. Rolipram promotes functional recovery after contusive thoracic spinal cord injury in rats. Behav Brain Res 2013; 243: 66-73.

17 Liang H, Li C, Gao A, Liang P, Shao Y, Lin T et al. Spinal duraplasty with two novel substitutes restored locomotor function after acute laceration spinal cord injury in rats. J Biomed Mater Res B App/ Biomater 2012; 100: 2131-2140.

18 Fehlings MG, Vaccaro A, Wilson JR, Singh A, Cadotte DW, Harrop JS et al. Early versus delayed decompression for traumatic cervical spinal cord injury: results of the Surgical Timing in Acute Spinal Cord Injury Study (STASCIS). PLOS ONE 2012; 7: e32037.

19 Girgis J, Merrett D, Kirkland S, Metz GA, Verge V, Fouad K. Reaching training in rats with spinal cord injury promotes plasticity and task specific recovery. Brain 2007; 130: 2993-3003.

20 Eftekharpour E, Karimi-Abdolrezaee S, Fehlings MG. Current status of experimental cell replacement approaches to spinal cord injury. Neurosurg Focus 2008; 24: E19.

21 Bai CB, Auerbach W, Lee JS, Stephen D, Joyner AL. Gli2, but not gli1, is required for initial shh signaling and ectopic activation of the shh pathway. Development 2002; 129: 4753-4761.

22 Kolterud A, Grosse AS, Zacharias WJ, Walton KD, Kretovich KE, Madison BB et al. Paracrine Hedgehog signaling in stomach and intestine: new roles for hedgehog in gastrointestinal patterning. Gastroenterology 2009; 137: 618-628.

23 Schnapp E, Kragl M, Rubin L, Tanaka EM. Hedgehog signaling controls dorsoventral patterning, blastema cell proliferation and cartilage induction during axolotl tail regeneration. Development 2005; 132 : 3243-3253.

24 Lee Y, Miller HL, Russell HR, Boyd K, Curran T, McKinnon PJ. Patched2 modulates tumorigenesis in patched 1 heterozygous mice. Cancer Res 2006; 66: 6964-6971.

25 Bambakidis NC, Onwuzulike K. Sonic hedgehog signaling and potential therapeutic indications. Vitam Horm 2012; 88: 379-394.

26 Machold R, Hayashi S, Rutlin M, Muzumdar MD, Nery S, Corbin JG et al. Sonic hedgehog is required for progenitor cell maintenance in telencephalic stem cell niches. Neuron 2003; 39: 937-950.

27 Berman DM, Karhadkar SS, Maitra A, Montes De Oca R, Gerstenblith MR, Briggs $\mathrm{K}$ et al. Widespread requirement for Hedgehog ligand stimulation in growth of digestive tract tumours. Nature 2003; 425: 846-851.

28 Karhadkar SS, Bova GS, Abdallah N, Dhara S, Gardner D, Maitra A et al. Hedgehog signalling in prostate regeneration, neoplasia and metastasis. Nature 2004; 431: 707-712.

29 Ochoa B, Syn WK, Delgado I, Karaca GF, Jung Y, Wang J et al. Hedgehog signaling is critical for normal liver regeneration after partial hepatectomy in mice. Hepatology 2010; 51: 1712-1723.

30 Belgacem YH, Borodinsky LN. Sonic hedgehog signaling is decoded by calcium spike activity in the developing spinal cord. Proc Natl Acad Sci USA 2011; 108: 4482-4487.

31 Barros Filho TE, Molina AE. Analysis of the sensitivity and reproducibility of the Basso, Beattie, Bresnahan (BBB) scale in Wistar rats. Clinics 2008; 63: 103-108.

32 Warzecha J, Gottig S, Bruning C, Lindhorst E, Arabmothlagh M, Kurth A. Sonic hedgehog protein promotes proliferation and chondrogenic differentiation of bone marrow-derived mesenchymal stem cells in vitro. J Orthop Sci 2006; 11: 491-496.

33 Jia Y, Wu D, Zhang R, Shuang W, Sun J, Hao H et al. Bone marrow-derived mesenchymal stem cells expressing the Shh transgene promotes functional recovery after spinal cord injury in rats. Neurosci Lett 2014; 573: 46-51.

34 Zhang J, Li Y, Zhang ZG, Lu M, Borneman J, Buller B et al. Bone marrow stromal cells increase oligodendrogenesis after stroke. J Cereb Blood Flow Metab 2009; 29: 1166-1174.

35 Bigelow RL, Chari NS, Unden AB, Spurgers KB, Lee S, Roop DR et al. Transcriptional regulation of $\mathrm{bcl}-2$ mediated by the sonic hedgehog signaling pathway through gli-1. J Biol Chem 2004; 279: 1197-1205

36 Du WZ, Feng Y, Wang XF, Piao XY, Cui YQ, Chen LC et al. Curcumin suppresses malignant glioma cells growth and induces apoptosis by 
inhibition of SHH/GLI1 signaling pathway in vitro and vivo. CNS Neurosci Ther 2013; 19: 926-936.

37 Zacharias WJ, Li X, Madison BB, Kretovich K, Kao JY, Merchant JL et al. Hedgehog is an anti-inflammatory epithelial signal for the intestinal lamina propria. Gastroenterology 2010; 138: 2368-2377 2377.e1-4.

38 Milenkovic L, Scott MP, Rohatgi R. Lateral transport of smoothened from the plasma membrane to the membrane of the cilium. J Cell Biol 2009; 187: 365-374.

39 Saada JI, Pinchuk IV, Barrera CA, Adegboyega PA, Suarez G, Mifflin RC et al. Subepithelial myofibroblasts are novel nonprofessional APCs in the human colonic mucosa. J Immunol 2006; 177: 5968-5979.

40 Rohatgi R, Milenkovic L, Scott MP. Patched1 regulates hedgehog signaling at the primary cilium. Science 2007; 317: 372-376.

41 Zhang Z, He Q, Deng W, Chen Q, Hu X, Gong A et al. Nasal ectomesenchymal stem cells: multi-lineage differentiation and transformation effects on fibrin gels. Biomaterials 2015; 49: 57-67.

42 Hou T, Wu Y, Wang L, Liu Y, Zeng L, Li M et al. Cellular prostheses fabricated with motor neurons seeded in self-assembling peptide promotes partial functional recovery after spinal cord injury in rats. Tissue Eng Part $A$ 2012; 18: 974-985.

(c) (i) This work is licensed under a Creative Commons Attribution 4.0 International License. The images or other third party material in this article are included in the article's Creative Commons license, unless indicated otherwise in the credit line; if the material is not included under the Creative Commons license, users will need to obtain permission from the license holder to reproduce the material. To view a copy of this license, visit http:// creativecommons.org/licenses/by/4.0/

(C) The Author(s) 2017 